Document downloaded from:

http://hdl.handle.net/10251/166518

This paper must be cited as:

Dhakshinamoorthy, A.; Asiri, AM.; García Gómez, H. (2020). Integration of metal organic frameworks with enzymes as multifunctional solids for cascade catalysis. Dalton Transactions. 49(32):11059-11072. https://doi.org/10.1039/d0dt02045a

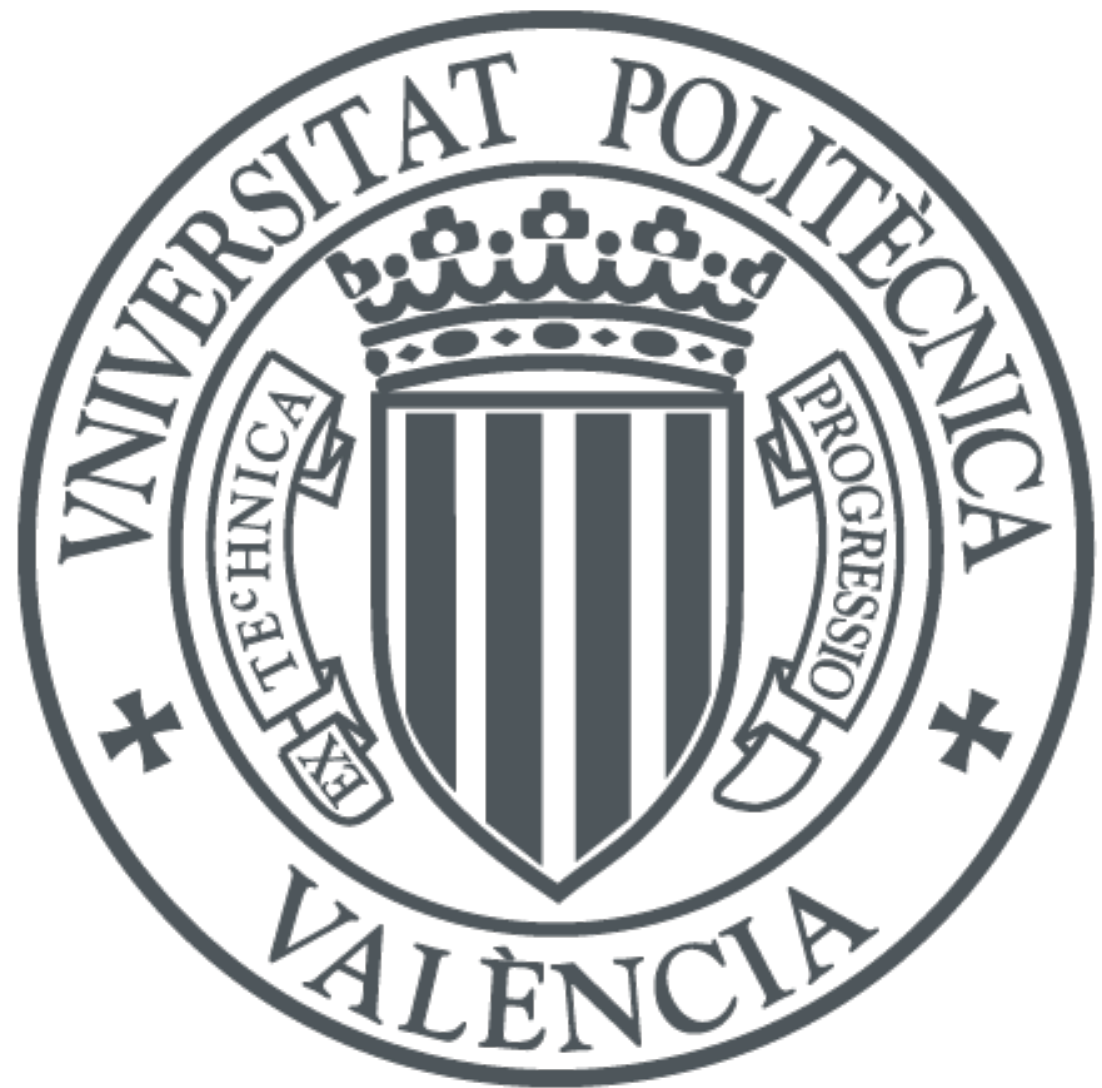

The final publication is available at

https://doi.org/10.1039/d0dt02045a

Copyright The Royal Society of Chemistry

Additional Information 


\title{
Integration of metal organic frameworks with enzymes as multifunctional solids for cascade catalysis
}

Amarajothi Dhakshinamoorthy, ${ }^{{ }_{a}}$ Abdullah M. Asiri, ${ }^{b}$ and Hermenegildo Garcia ${ }^{* b, c}$

\author{
aSchool of Chemistry, Madurai Kamaraj University, Madurai-625 021, Tamil Nadu, India. \\ E-mail: admguru@gmail.com \\ ${ }^{b}$ Center of Excellence for Advanced Materials Research, King Abdulaziz University, Jeddah, Saudi \\ Arabia \\ 'Departamento de Quimica and Instituto Universitario de Tecnologia Quimica (CSIC-UPV), Universitat \\ Politecnica de Valencia, Av. De los Naranjos s/n, 46022 Valencia, Spain. E-mail: hgarcia@qim.upv.es
}

\begin{abstract}
Enzymes exhibit a large degree of compatibility with metal-organic frameworks (MOFs) that allow to develop multicomponent catalysts constituted by enzymes adsorbed or occluded by MOFs. The combination of enzymes and MOFs in a multicomponent catalyst can be used to promote cascade reactions in which two or more individual reaction are performed in a single step. The cascade reactions take place due to the cooperation of active sites present on the MOF with the enzyme. Survey of the available data establishes that often the enzyme undergoes stabilization by association with MOF and the system exhibits a notable recyclability. In addition, the existence of a synergism is observed as consequence of the close proximity of all the required active sites in the multicomponent catalyst. After an introductory section describing the specific features and properties of enzyme-MOF assemblies, the main part of the present review focuses on the description of the cascade reactions that have been reported with commercial enzymes associated with MOFs, paying special attention to the advantages derived from the multicomponent catalyst. Related with the catalytic activity to metabolize glucose, generating reactive oxygen species and decreasing the solution $\mathrm{pH}$, an independent section describes the recent use of enzyme-MOF catalysts in cancer therapy. The last paragraphs summarize the current state of the art and provide our view on future developments in the field.
\end{abstract}

\section{Introduction}


Enzymes are the most efficient and selective catalysts working under mild, physiological conditions. ${ }^{1}$ The use of enzymes to synthesise organic molecules, particularly for the commercial production of complex multifunctional compounds of interest as pharmaceuticals has been growing continuously. Under metabolic conditions, transformation of biomolecules requires the cooperative contribution of several enzymatic systems, resulting in the occurrence of various individual reactions in which the product of one transformation is the substrate for the subsequent process. ${ }^{2-4}$ These types of consecutive reactions, combining in a single step two or more different reactions, are generally known as cascade or tandem reactions and they represent an example of process intensification. ${ }^{5-7}$ Cascade reactions minimize tedious reaction work-up and make unnecessary isolation of intermediate compounds in a synthetic route.

The complexity of biochemical cycles involving several enzymatic systems makes difficult to mimic the cascade processes occurring in the synthesis of biomolecules in a non-natural environment. However, a step forward is to couple enzymatic activity with conventional acid/base or redox catalysis due to transition metal sites.8, 9 Particularly interesting is when heterogeneous solid catalysts are combined with enzymes that remain adsorbed or occluded within the solid, since in this way, the enzyme can become immobilized and can be easily separated from the reaction mixture and recycled. ${ }^{10-}$ 12

While enzymes have been attached to a large variety of carbon materials or high surface area metal oxides, there is a growing interest in the use of MOFs as supports and complementary catalysts for enzymes. ${ }^{13,14}$ The main reason for this interest is the synergy that has been often observed for this type of multicomponent MOF catalysts compared to alternative supports.

MOFs are porous crystalline coordination polymers whose structure is constituted by nodes of metal ions or clusters of metal ions in strong interaction through Coulombic and coordinative bonds with rigid multipodal organic ligands. ${ }^{15-17}$ The defined directionality of coordinative bonds around the metal nodes and the geometry of the binding sites of the organic ligand determine a low density of the unit cell that has an open porosity in the range of micro- and mesoporosity. MOFs are materials with a very large surface area and pore volume. ${ }^{18-21}$ The reader is referred to the existing literature for an in-depth description of the synthesis, structures, physical properties and applications of MOFs, including their use as heterogeneous catalysts. ${ }^{10,11,22-25}$ 
In the literature there are basically three possible strategies to obtain the enzyme-MOF composite (Chart 1). One of them requires MOFs of pore sizes larger than the enzyme dimension. ${ }^{11,26 \text {, }}$ ${ }^{27}$ Enzymes have a wide range of molecular weight and sizes, but many of them have dimensions in the mesoporous scale between 3 to $6 \mathrm{~nm}$. Thus, for certain large-pore MOFs it could be possible to incorporate enzymes within their mesopores. However, large pore MOFs tend to be remarkably instable and this strategy is limited to only a few MOF structures.

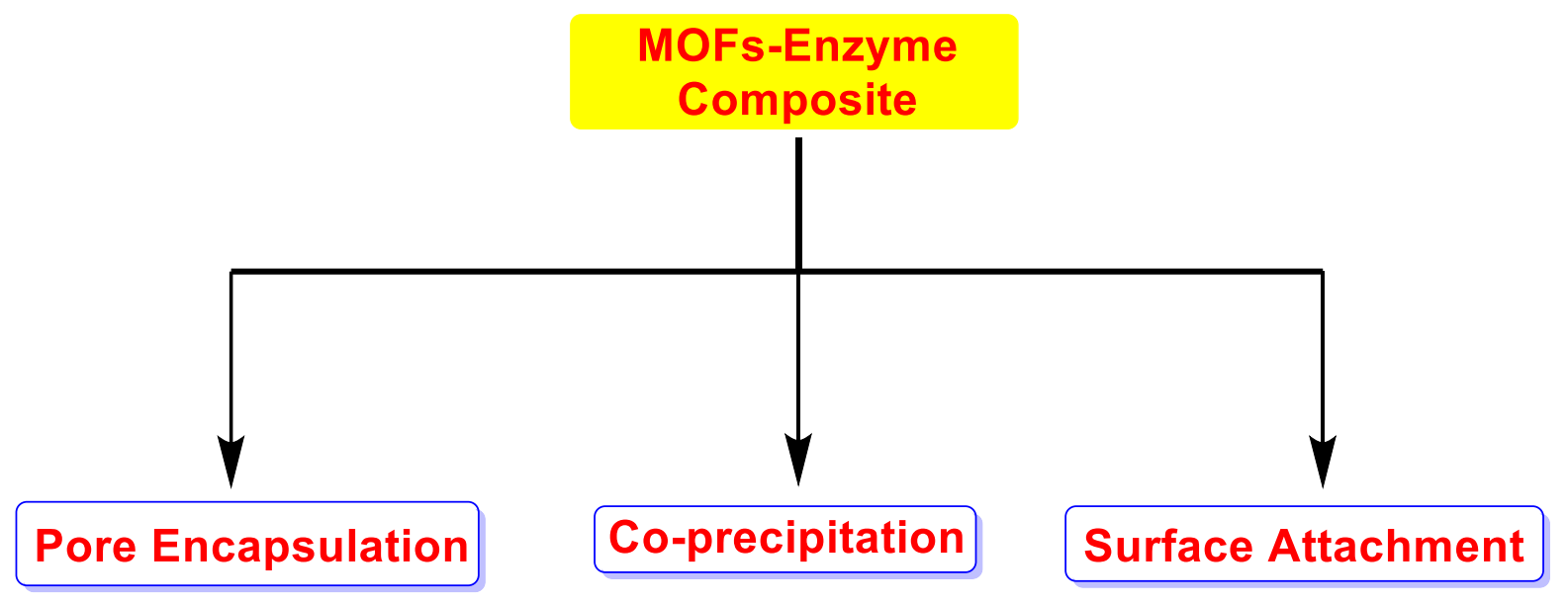

Chart 1. Most commonly employed strategies for the preparation of MOFs-enzyme composite.

The most common and robust MOFs have pore sizes about 1-2 nm, therefore, too small to accommodate enzymes within their voids. However, even in these cases, MOFs crystallites can be formed around the enzyme that becomes the core of the material surrounded by a dense layer of MOF particles. ${ }^{11,} 25,28$ The inner enzyme will still be accessible to substrates due to MOF porosity. The requisite in this strategy is the compatibility of MOF synthesis with enzyme stability. This limits the range of MOFs that can be used to those whose synthesis is mainly a precipitation of the metal ions by the ligands, without the need of lengthy hydrothermal treatment in organic solvents at high temperatures. In this strategy the functional groups of the enzyme can adsorb metal ions, initiating the nucleation of the MOF.

In a third strategy, enzyme becomes adsorbed onto the external surface of MOF microcrystals. In some cases, the enzyme-MOF association is strong since it is due to Coulombic interactions due to complementary surface charges of both components. ${ }^{9,11}$ 
Besides, design capability and large compositional and structural diversity, one advantage of using MOFs as support for enzymes are the presence of active sites. Active sites in MOFs can be due to i) the presence of Lewis acid sites in exchangeable coordination positions around the transition metals, ii) substituents on the organic ligands, iii) the presence of structural defects, and iv) the presence of active guests within the pores, such as metal nanoparticles (NPs) or metal complexes. As it will be shown in the following sections, this range of possibilities can be combined with the enzymatic activity, developing stable, recyclable, enzyme-MOF catalysts for cascade reactions.

For obvious reasons of availability, the current existing literature on enzyme-MOF catalysts for cascade reactions is mostly limited to commercially available enzymes, such as lipases and oxidases. However, it will be emphasised in the sequel that there are sufficient number of examples showing the enzymes are largely compatible with the composition and structure of MOFs. Even more, there is evidence showing that the enzyme becomes stabilized and its activity maintained as consequence of its immobilization within/on MOFs. In addition, examples of synergy between the two components as consequence of their association and close proximity has been also frequently observed by comparing the activity of the multicomponent enzyme-MOF catalyst compared to their independent components. ${ }^{11}$ It appears that the close spatial proximity among the various active sites involved in the cascade process favours a faster diffusion of the intermediates, resulting in a faster reaction and, consequently, in a higher selectivity derived from the formation of by-products in a lesser percentage.

The main part of the review describes relevant examples of the catalytic activity of enzymeMOF catalyst in cascade reaction for the production of chemicals or for reaction with biomolecules not only for the synthesis of products by also for sensing application. Those reports describing the cooperation of more than one enzyme will also be described. In a separate section the possibility to use enzyme-MOF systems for cancer therapy by causing tumoral cell death by starvation and over production of reactive oxygen species will also presented. The last section of the review will summarize the main points of the current state of the art and will provide our prospects for future development of the field.

\section{Enzymes@MOFs for cascade catalysis}

One long standing goal in the development of multifunctional solid catalysts is the rational engineering and assembly of diverse active centres in a highly structured material to achieve high 
reactivity and selectivity. In this respect, ultra-large mesopores $(20-40 \mathrm{~nm})$ MOF crystals have been prepared by competitive coordination chemistry with polyvinylpyrrolidone (PVP), allowing the incorporation of large guests, like enzymes whose dimensions are tens of $\mathrm{nm}$. The resulting composite integrates different catalytic sites such as Lewis acids through coordinatively unsaturated metal sites of the nodes, possible occluded metal nanocrystals (NCs) and enzymes in a single MOF solid (Figure 1). ${ }^{29}$

In one example of this strategy, a porous, defect-rich ZIF-67 (DP-ZIF67) (ZIF: Zeolitic Imidazolate Framework) was obtained employing PVP as metal coordinating bulky polymer during the crystallization of ZIF-67. The presence of PVP induces partial disruption of imidazole-Co(II) catenation, resulting in the generation of defects, and, simultaneously, creates high mesoporosity in DP-ZIF67 crystals. Interestingly, transmission electron microscopy (TEM) images revealed that DP-ZIF67 has identical rhombic dodecahedron morphology as pristine ZIF-67 with particles ca. $800 \mathrm{~nm}$ size. Brunauer-Emmett-Teller (BET) surface area measurements indicated DP-ZIF67 and ZIF-67 exhibit 1654 and $1672 \mathrm{~m}^{2} \mathrm{~g}^{-1}$, respectively. X-Ray photoelectron spectroscopy (XPS) analysis revealed a twofold increase in the density of unsaturated Co sites from $7.8 \%$ for ZIF-67 to $18.3 \%$ for DP-ZIF67. Pd NCs were loaded on DP-ZIF67 to obtain Pd@DP-ZIF67, which was further incubated with Candida antarctica lipase A (CalA) to provide solid Pd@DP-ZIF67/CalA integrating the enzyme. CalA is an enzyme able to promote enantioselective esterification, particularly acetylations using enol acetates as reagents. The loading of enzyme in the final solid was $26.5 \%$ as evidenced by the colorimetric Bradford assay. The Fourier-transform infrared (FT-IR) spectrum of Pd@DP-ZIF67/CalA exhibited C=O stretching vibration at $1660 \mathrm{~cm}^{-1}$ corresponding to amide-I of the enzyme, in addition to other characteristic peaks for ZIF-67.

The catalytic efficiency of Pd@DP-ZIF67/CalA was assessed in the cascade reaction involving the nitroaldol reaction promoted by the Lewis acid sites, Pd-NCs as racemisation catalyst and CalA for enzymatic kinetic resolution and acylation. Under the optimized reaction conditions, the reaction between $\mathrm{p}$-anisaldehyde and nitromethane in $\mathrm{N}, \mathrm{N}$-diisopropylethylamine and vinylacetate as the acyl donor using Pd@DP-ZIF67/CalA at room temperature afforded the desired chiral acetylated nitroalcohol with $>97 \%$ conversion and $>99 \%$ enantiomeric excess ( $>99 \%$ ee) (Figure $2 a)$. In contrast, a series of control experiments with a mixture of $\mathrm{Co}\left(\mathrm{NO}_{3}\right)_{2} \cdot 6 \mathrm{H}_{2} \mathrm{O}$ and imidazole as well as $\mathrm{ZIF}-67$ showed very poor activities compared to the DP-ZIF67 (Figure 2b). On other hand, DP-ZIF67 pre- 
treated with excess 2-methylimidazole (2-Melm) exhibited poor yield of the nitroaldol product due to the poisoning of unsaturated $\mathrm{Co}$ (II) sites by saturation, indicating the effective role of Lewis acid sites. Lower activities in the formation of the nitro acetate product were also attained for the physical mixture of DPZIF67, Pd NCs and CalA (ca. 20\% ee) as well as the physical mixture of Pd@DP-ZIF67 and CalA@DPZIF67 (ca. 38\% ee). In another control experiment, the physical mixture of Pd@DP-ZIF67 and free CalA was employed as catalysts under identical conditions, observing only ca. $24 \%$ ee of the product. These control experiments clearly show the superior performance of the integrated Pd@DP-ZIF67/CalA solid catalyst, indicating clearly the effect of immobilization of CalA within the mesopores of MOFs not only by increasing the stability, but reaching higher activity through the confinement effect due to the close intimacy between the active sites.

Pd@DP-ZIF67/CalA was facilely recovered and reused in five successive cycles with $86 \%$ yield and $>80 \%$ ee of product. Further, TEM, high resolution transmission electron microscopy (HRTEM) and energy dispersive spectroscopy (EDS) mapping analyses indicated that no significant change in the particle morphology, Pd NC particle size and CalA loading takes place in five cycles. However, the slight decrease in the activity was due to Pd leaching as evidenced by inductively coupled plasma (ICP) analysis. Interestingly, Pd@DP-ZIF67/CalA exhibited very high yields (>99\%) and ee (>99\%) of various nitro acetates from different aryl aldehydes irrespective of the electron donating or withdrawing nature of the substituents.

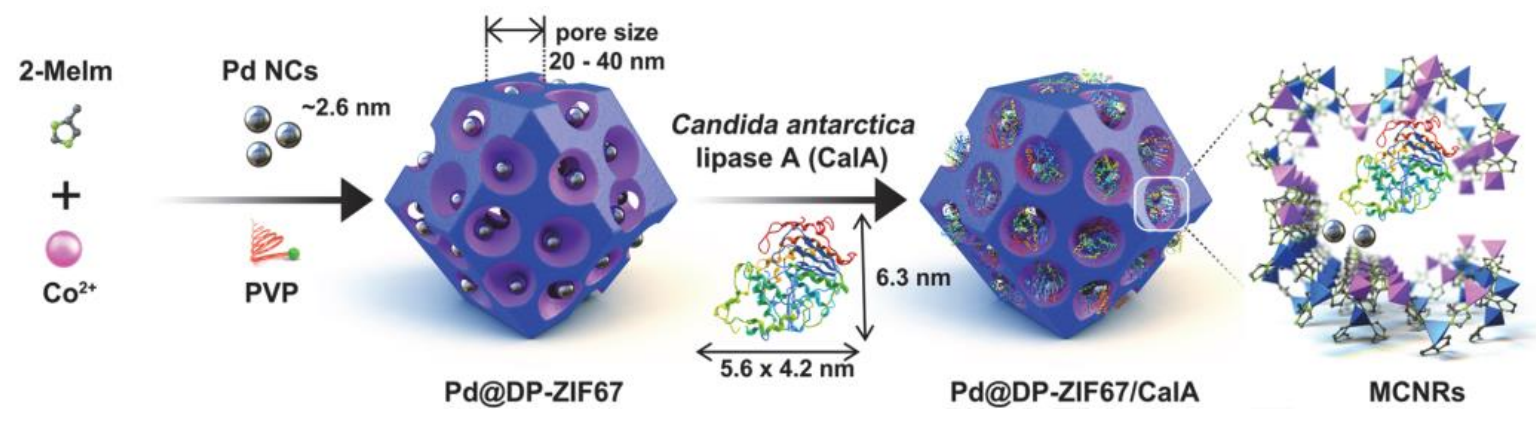

Figure 1. Synthetic strategy towards multimodal catalytic nanoreactors (MCNRs) containing coordinatively unsaturated $\mathrm{Co}^{2+}$ (violet colored metal sites), Pd NCs and CalA inside DP-ZIF67. Reproduced with permission from ref. ${ }^{29}$ Wiley, Copyright 2020. 

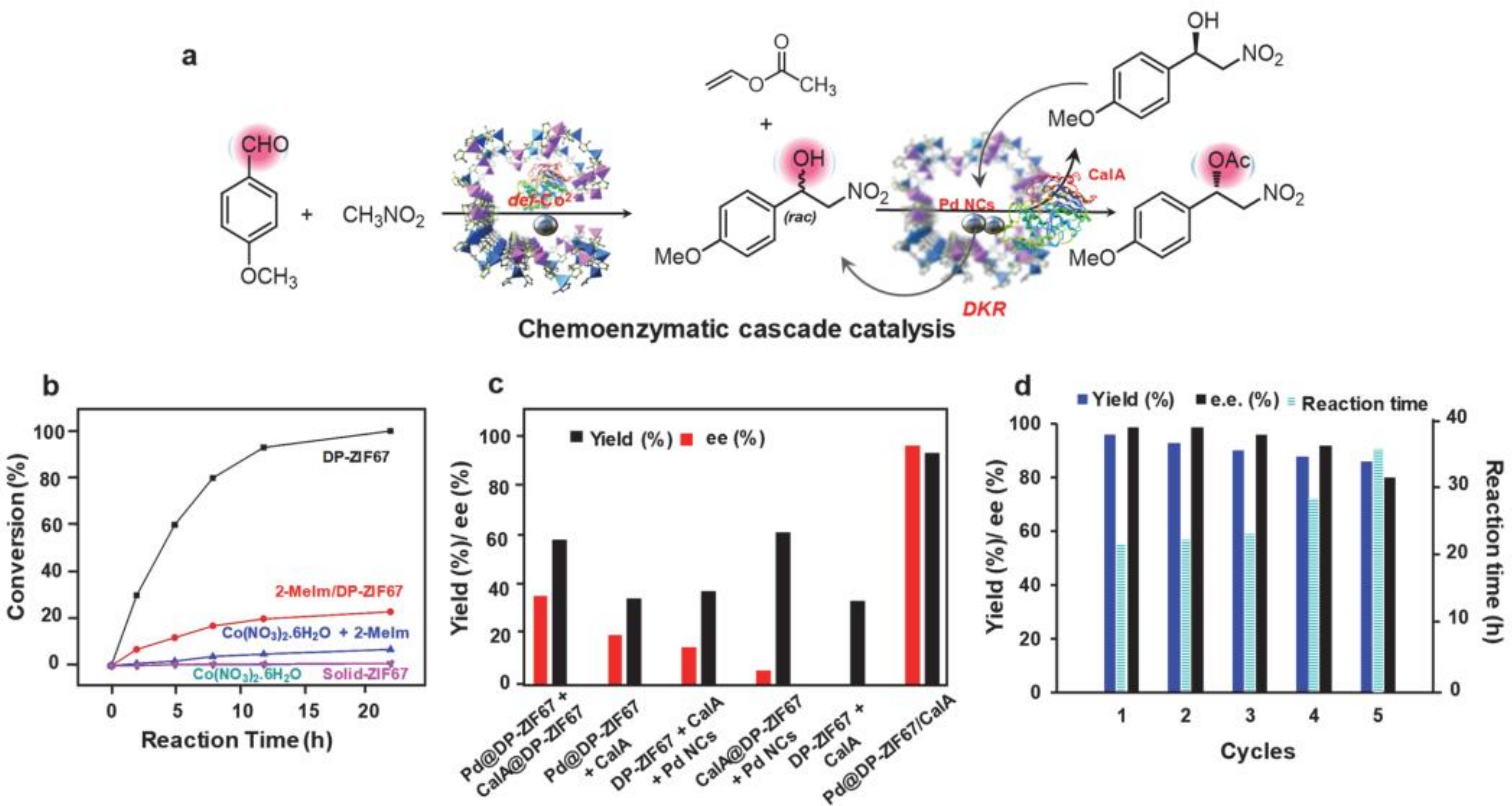

Figure 2. (a) Schematic illustration of the cascade reaction catalyzed by Pd@DP-ZIF67/CalA, (b) Kinetics of nitrolaldol reaction catalyzed by DP-ZIF67 and various control catalysts. (c) The Pd@DPZIF67/CalA and various control catalysts catalyzed cascade catalysis to obtain enantiomerically pure $\beta$-nitroacetate, in high yield showing that co-immobilization of the active sites has a positive effect on the product ee and product yield. (d) Reaction yield, ee values and time in five cycles for the conversion of $p$-anisaldehyde to $\beta$-nitroacetate using Pd@DP-ZIF67/CalA as catalyst. Reproduced with permission from ref ${ }^{29}$ Wiley, Copyright 2020.

An amino functionalized UiO-66 MOF $\left(\mathrm{UiO}-66-\mathrm{NH}_{2}\right)$ was subjected to ligand exchange by lauric acid (LA) to obtain a sample denoted as UiO-LA, which was further used as a robust host for the stepwise immobilization of Pd (NPs) and Candida antarctica lipase B (CalB) to obtain a multicomponent catalyst (CalB-Pd@UiO-LA; Figure 3). The catalytic activity of this solid was tested in the synthesis of benzyl hexanoate from benzaldehyde and ethyl hexanoate through cascade reactions. ${ }^{30}$ The average diameter of Pd NPs was around $3.41 \pm 0.49$ nm. Among the various catalysts screened, CalB-Pd@UiOLA50 solid afforded 100\% yield of benzyl hexanoate after 8 h, while either CalB-Pd@UiO or CalBPd@UiO-LA20 provided 76\% yield under identical conditions (Scheme 1). The superior performance of CalB-Pd@UiO-LA50 was ascribed to its improved dispersability in the reaction medium thus facilitating the interaction of substrates with the active sites. In contrast, the activity of the physical mixture of Pd@UiO-LA50 and free CalB was lower than CalB-Pd@UiO-LA50, a result that was attributed to the aggregation of free CalB. The yield of benzyl hexanoate was retained at about $80 \%$ 
after three cycles. This minor decrease in the activity is due to Pd leaching, while no obvious leaching of CalB was observed as assessed by Bradford test.

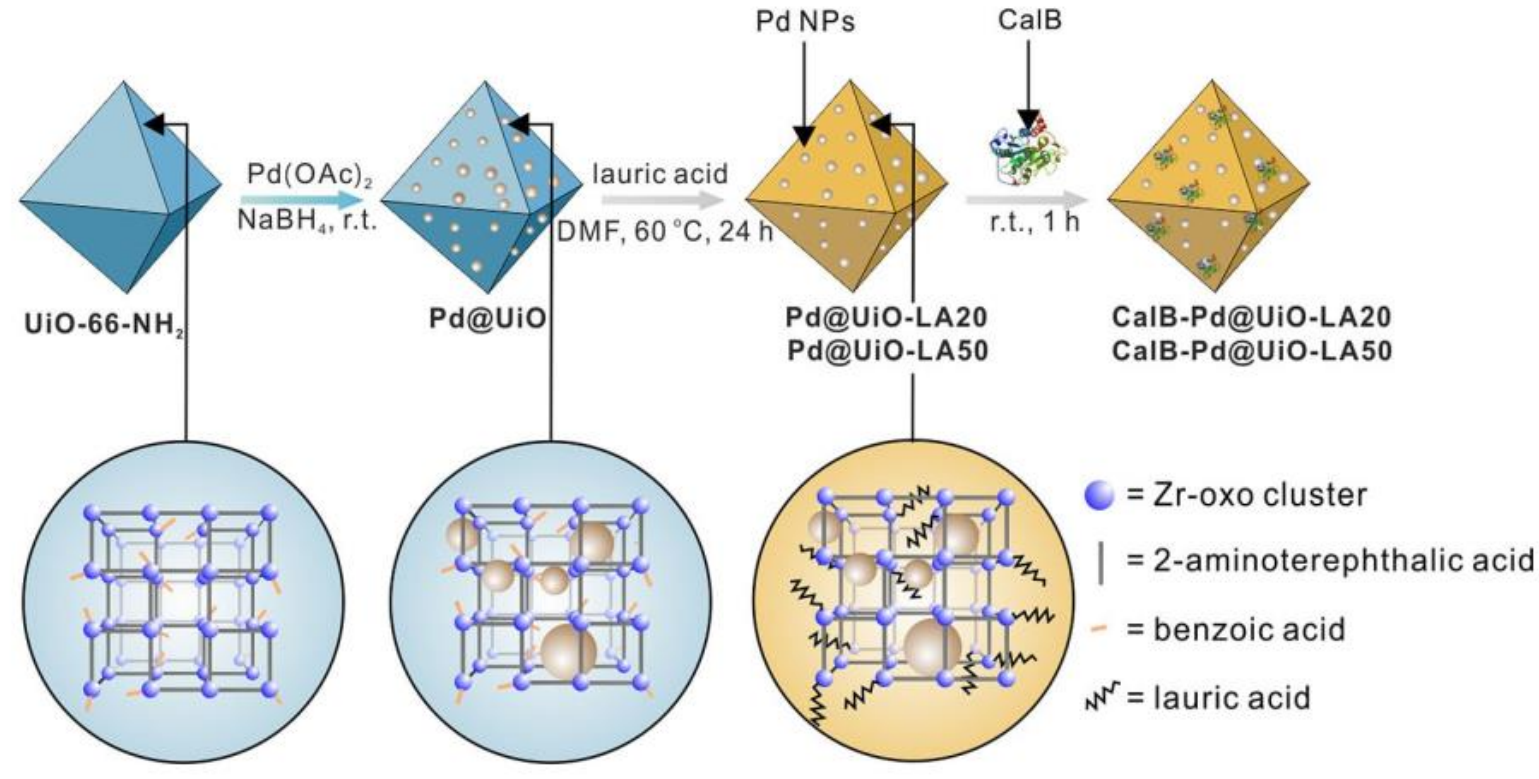

Figure 3. Synthesis of CalB-Pd@UiO-LA20 and CalB-Pd@UiO-LA50. Reproduced with permission from ref. ${ }^{30}$ Wiley, Copyright 2019.

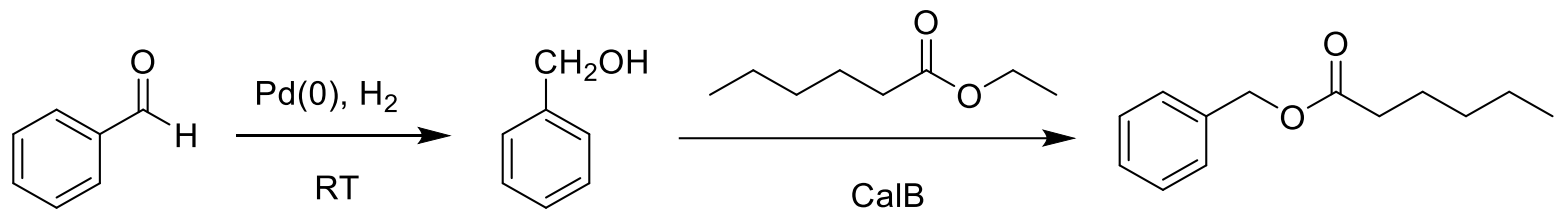

Scheme 1. Synthesis of benzyl hexanoate from benzaldehyde through cascade catalysis by CalBPd@UiO-LA50.

\section{Enzymes@MOFs for cascade biocatalysis}

\section{1 Encapsulation of mono enzymes}

Another commercially available enzyme that has been widely used in combination with MOFs is glucose oxidade (GOx) that catalyzes the selective aerobic oxidation in aqueous media of glucose to gluconic acid. A self-activated MOF/enzyme hybrid nanoreactor (MIL@GOx-MIL NRs) was fabricated by employing MIL-101(Fe)-NH 2 NPs and GOx through a two-step procedure (Figure 4) ${ }^{31}$ In this catalytic system, GOx is responsible for the conversion of glucose to gluconic acid, a conversion that lowers the pH from 7.4 to around 4. Further, MIL@GOx-MIL NRs afforded the highest performance in hydroxyl 
radical generation from $\mathrm{H}_{2} \mathrm{O}_{2}$ at $\mathrm{pH} 4$ than at $\mathrm{pH}$ 7.4. Therefore formation of gluconic acid activates the efficient generation of hydroxyl radicals.

Furthermore, MIL@GOx-MIL NRs exhibits an interesting antibacterial activity. This biocide activity was studied by a standard plate counting assay, whereby it was observed that the growth of methicillin-resistant staphylococcus aureus (MRSA) is effectively limited at $5 \mu \mathrm{g} / \mathrm{mL}$ of MIL@GOx-MIL NRs. On other hand, nearly no MRSA biofilms were seen at a dosage of $80 \mu \mathrm{g} / \mathrm{mL}$ of MIL@GOxMIL NRs. Therefore, these results clearly illustrate the effective role of self-activated cascade catalysis in the field of combating drug-resistant bacteria and related disinfection of other pathogens.

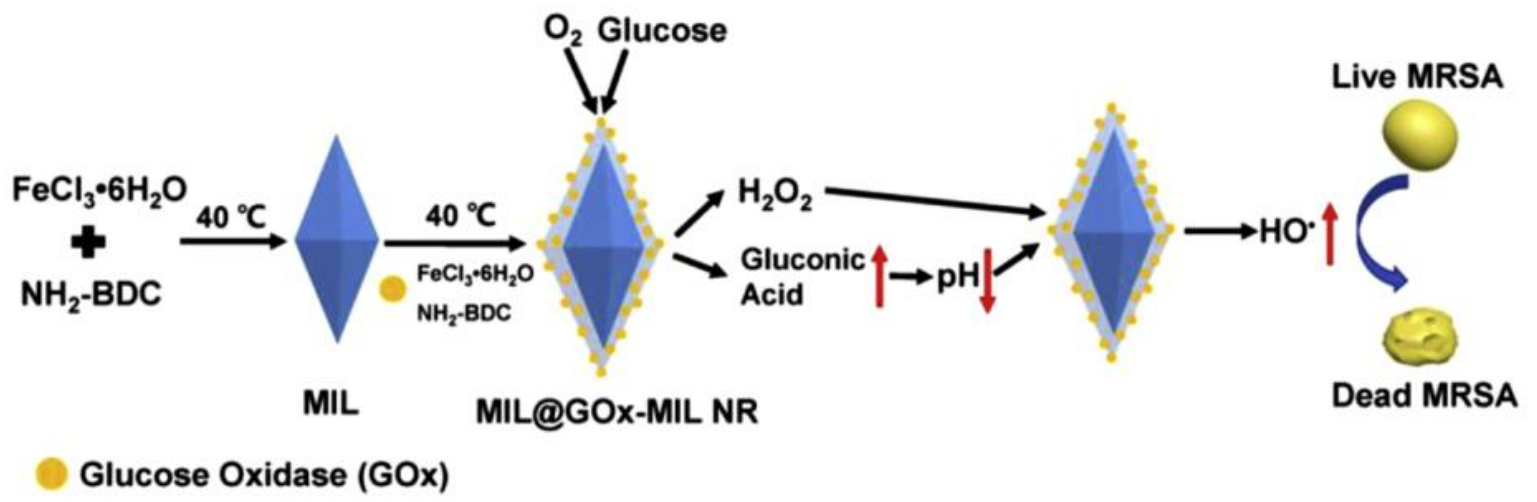

Figure 4. The fabrication of self-activated MOF/enzyme hybrid NRs based on MIL NPs and GOx and the mechanism on combating MRSA. Reproduced with permission from ref. ${ }^{31}$ Elsevier, Copyright 2020.

An amino functionalized Fe-MOF (Fe-MIL-88B-NH $\mathrm{NH}_{2}$ was employed as support for the immobilization of GOx assisted by cross-linking agents and the resulting composite (Fe-MOF-GOx) activity was examined in the cascade reaction involving oxidation of glucose followed by $3,3^{\prime}, 5,5^{\prime}$ tetramethylbenzidine (TMB) (Figure 5) ${ }^{32}$ The experimental results have shown that Fe-MOF-GOx exhibited 1.4 fold increased absorbance than the physical mixture of Fe-MOF and GOx under identical conditions. This enhanced activity of Fe-MOF-GOx is ascribed to the confinement of reactants within the nanometric reactors diminishing diffusion limitations and minimizing the decomposition of $\mathrm{H}_{2} \mathrm{O}_{2}$ generated from the GOx-catalyzed oxidation of glucose with $\mathrm{O}_{2}$. Furthermore, the catalytic activity of Fe-MOF-GOx was retained for five cycles, while the activity of Fe-MOF/GOx decreased sharply under identical conditions (Figure 6). 

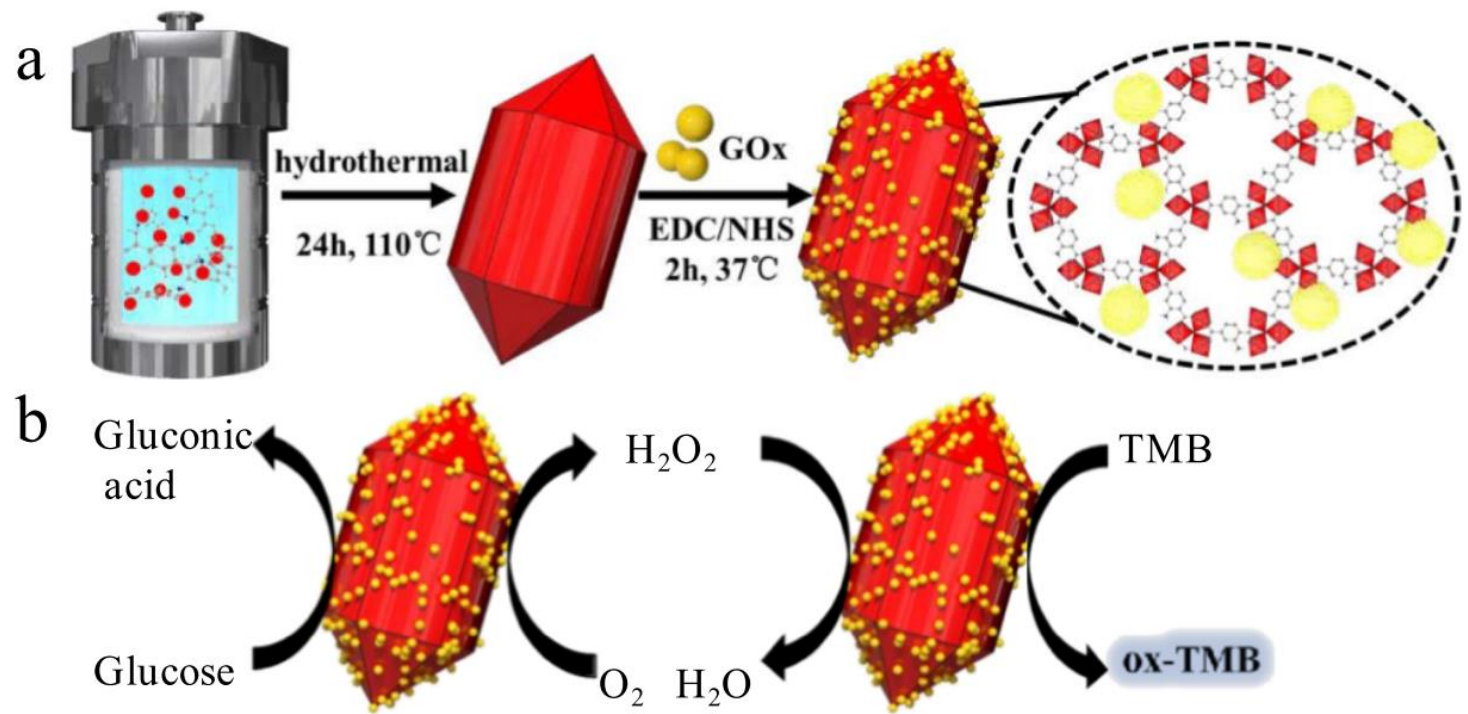

Figure 5. (a) Illustration of the synthetic approach of Fe-MOF-GOx and (b) corresponding cascade catalytic detection of glucose by Fe-MOF-GOx. Reproduced with permission from ref. ${ }^{32}$ American Chemical Society, Copyright 2019.

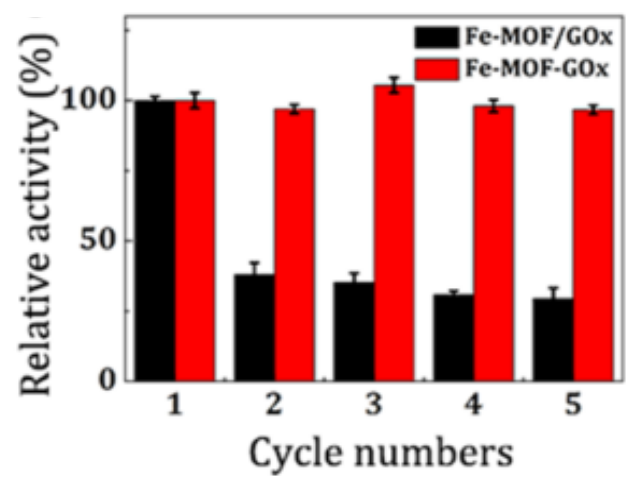

Figure 6. Relative catalytic activity of Fe-MOF/GOx and Fe-MOF-GOx in five successive cycles. Reproduced with permission from ref. ${ }^{32}$ American Chemical Society, Copyright 2019.

Later, PCN-222(Fe) (PCN: Porous Coordination Network) MOF was assembled by the reaction of highly stable $\mathrm{Zr}_{6}$ clusters with TCPP(Fe) (TCPP: tetrakis(4-carboxyphenyl)-porphyrin). Further, PCN222(Fe) exhibited strong electrostatic interaction with GOx which favoured negligible enzyme leaching. Hence, a facile strategy was reported for the immobilization of GOx on PCN-222(Fe) to obtain [GOx/PCN-222(Fe)] through Coulombic interactions in which PCN-222(Fe) serves as both a support and chemocatalyst (Figure 7). The cascade catalytic activity of GOx/PCN-222(Fe) was tested in the 
glucose oxidation and 2,2'-azino-bis(3-ethylbenzothiazoline-6-sulfonic acid (ABTS) or pyrogallol oxidation (Scheme 2). ${ }^{33}$ The kinetic experiments have clearly shown that the Michaelis constant $\left(\mathrm{K}_{\mathrm{m}}\right)$ for the oxidation of pyrogallol (0.72) is lower than that of ABTS oxidation (1.25). These catalytic data suggest that GOx shows higher affinity for pyrogallol due to its smaller size and easier diffusion inside the pores compared to bulkier ABTS. Furthermore, GOx/PCN-222(Fe) displayed identical conversion rate in six repeated cycles, thus showing the stability of this catalyst under these experimental conditions.

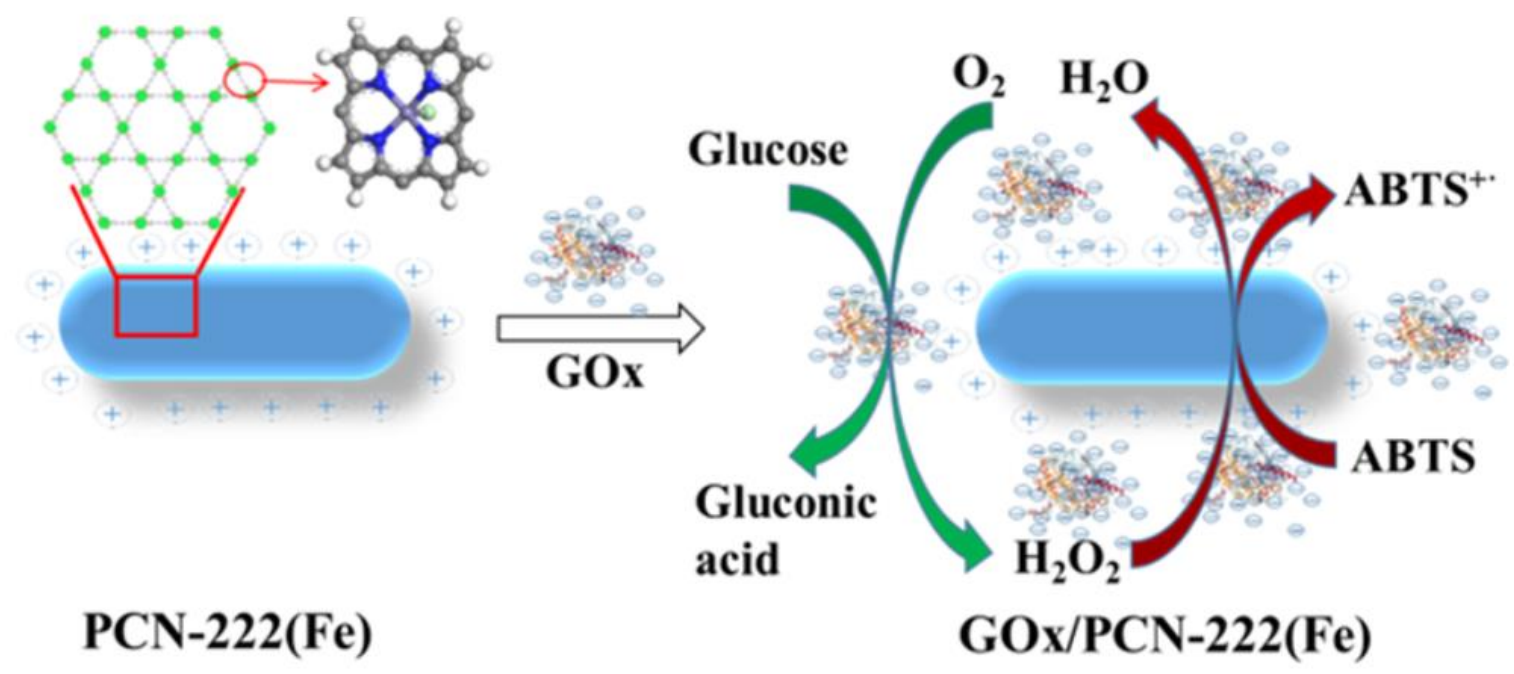

Figure 7. Immobilization of $\mathrm{GOx}$ on $\mathrm{PCN}-22(\mathrm{Fe})$ and cascade reaction involving glucose aerobic oxidation with generation of $\mathrm{H}_{2} \mathrm{O}_{2}$ and subsequent generation of ABTS radical cation. Reproduced with permission from ref. ${ }^{33}$ American Chemical Society, Copyright 2019. 
a)

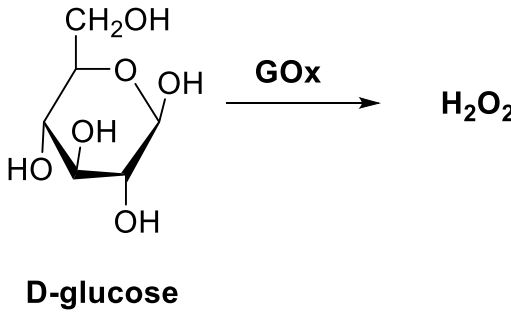

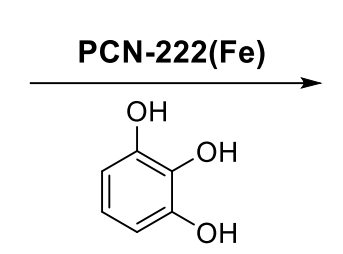

pyrogallol<smiles>O=c1c(O)cccc2cc(O)c(O)c(O)c12</smiles>

purpurogallin b)

D-glucose

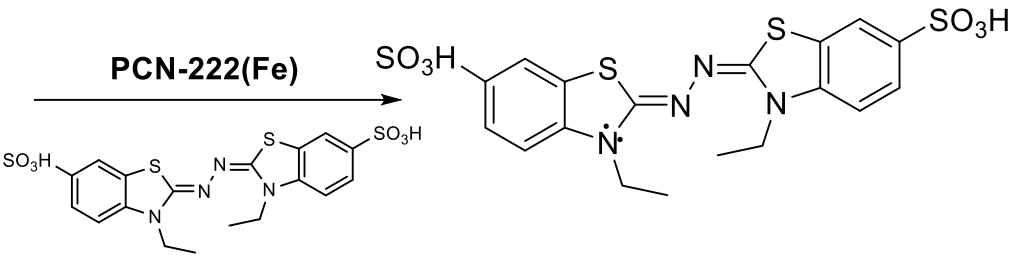

ABTS

ABTS . $^{+}$

Scheme 2. Oxidation of pyrogallol and ABTS by GOx/PCN-222(Fe) by coupling $\mathrm{H}_{2} \mathrm{O}_{2}$ generation and enzymatic glucose oxidation.

In another work, the peroxidase-like activity of hierarchically porous MOF, HP-PCN-224(Fe) allowed the design of a cascade catalyst by immobilizing GOx over HP-PCN-224(Fe) to obtain GOx@HP-PCN-224(Fe) (Figure 8). ${ }^{34}$ Among the various experimental conditions tested, in more acidic or alkaline conditions, GOx@HP-PCN-224(Fe) exhibited superior activity compared to free GOx. Further, GOx@HP-PCN-224(Fe) maintained about 90 \% residual activity after five cycles, showing excellent stability under these conditions. 


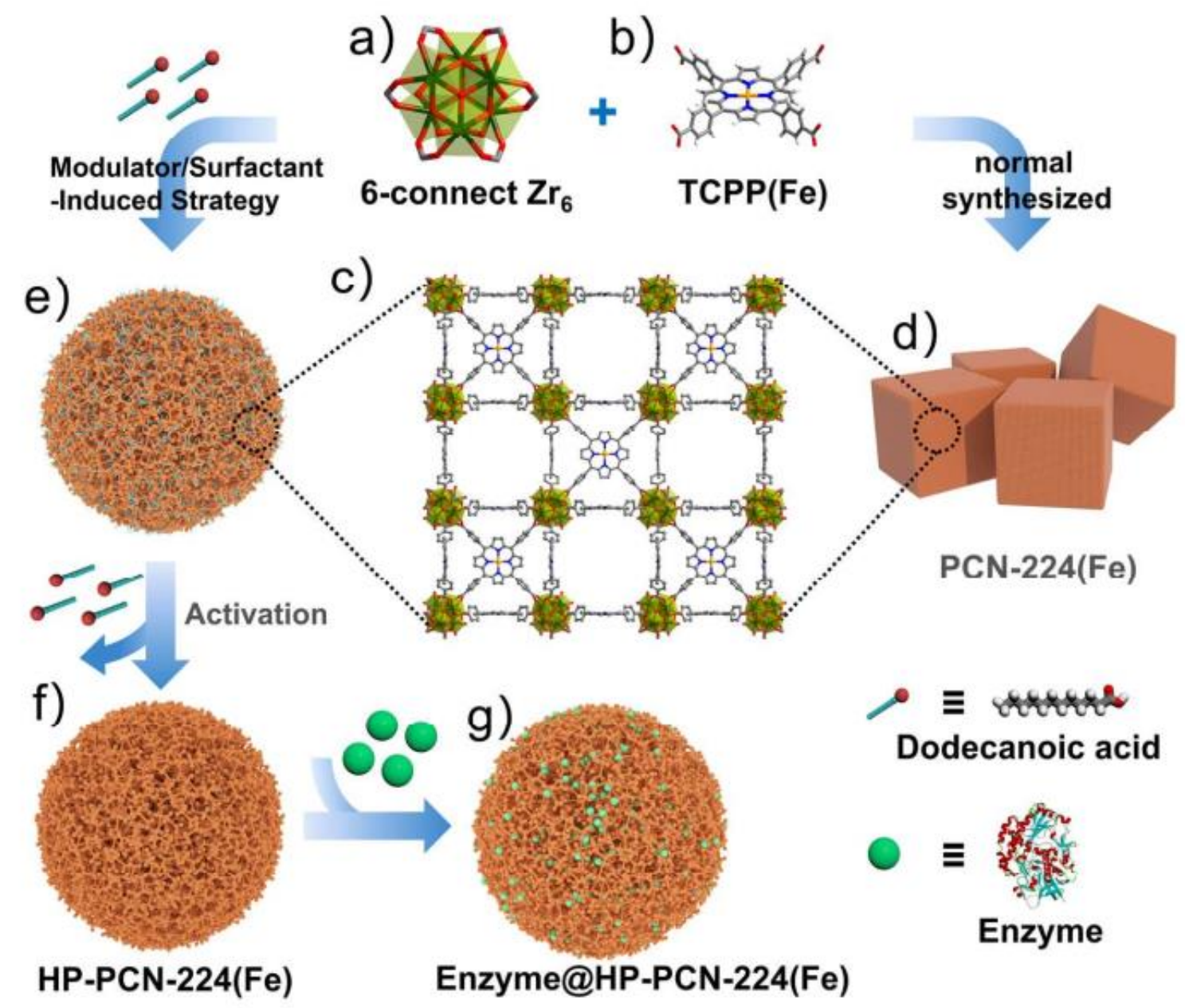

Figure 8. Schematic diagram of the preparation of the mimic multi-enzyme system enzyme@HP-PCN224(Fe): (a) the 6-connected $\mathrm{D}_{3 \mathrm{~d}}$ symmetric $\mathrm{Zr}_{6}$ and (b) tetratopic TCPP ligand in PCN-224(Fe). (c) Crystal structure of PCN-224(Fe). (d) Schematic of the parent PCN-224(Fe). (e) The synthesis of hierarchically porous $\mathrm{PCN}-224(\mathrm{Fe})$ with a modulator/surfactant-induced strategy, $(\mathrm{f})$ the activation of hierarchically porous PCN-224(Fe), and (g) the mimic multi-enzyme systems. Reproduced with permission from ref. ${ }^{34}$ American Chemical Society, Copyright 2018.

In related study, a mimic multi-enzyme system was fabricated by immobilizing GOx within the pores of MOF-545(Fe) and the activity of the resulting enzymatic system (Figure 9) was evaluated in the cascade catalysis of the aerobic oxidation of glucose and ABTS radical cation generation. ${ }^{35}$ The visual observation of $\mathrm{ABTS}^{+\cdot}$ radical cation allows to apply the system as a glucose sensor. Thus, GOx@MOF-545(Fe) can serve for a rapid detection of glucose with a low limit of detection $(0.28 \mu \mathrm{M})$ and high specificity. Furthermore, GOx@MOF-545(Fe) retained 92\% of its original activity after stored 
for seven days, while only $40 \%$ of activity of free enzyme was maintained under analogous conditions.

On other hand, GOx@MOF-545(Fe) was used for five cycles by maintaining 71\% of its initial activity.
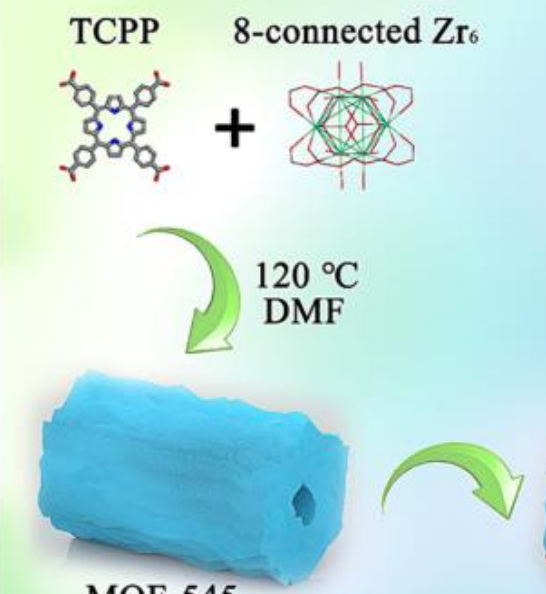

MOF-545

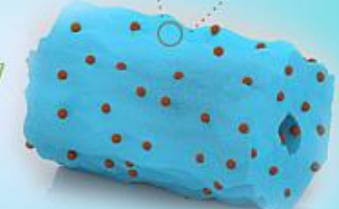

MOF-545 (Fe)
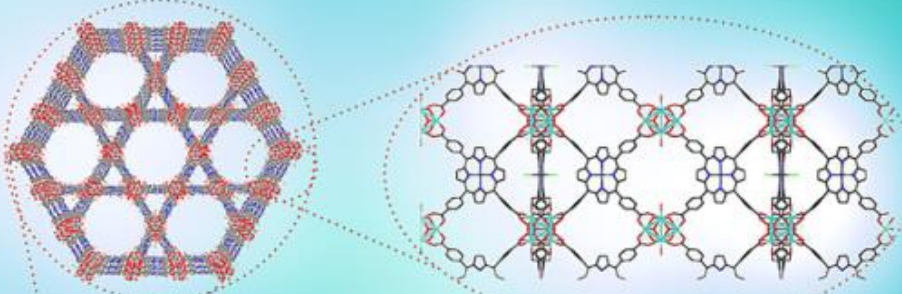

Fe: • Enzyme:

Figure 9. Schematic illustration of the preparation of GOx@MOF-545(Fe) composites through electrostatic surface adsorption. Reproduced with permission from ref. ${ }^{35}$ Elsevier, Copyright 2020.

\subsection{Encapsulation of dual enzymes}

Cascade reactions involving enzyme as one of the active sites have received considerable attention due to the exquisite substrate selectivity of the enzyme that makes possible to perform concurrent chemical reactions with high activity and selectivity. ${ }^{36}$ In this context, a so-called amino acidboosted one pot embedding (AAOPE) strategy was developed for the encapsulation of a broad range of proteins or enzymes within the pores of MOFs. ${ }^{37}$ The inspiration for the development of this strategy stemmed from the metal cations-accumulating pattern of the metallothioneins (Figure 10A) ${ }^{38}$ Hence, the proteins assembled with abundant cysteine (Cys) favoured the accumulation of $\mathrm{Zn}^{2+}$ ions by mercaptide bonds, and thus accelerated the formation of prenucleated clusters, allowing the fabrication of MOFs around the proteins (Figure 10B). One unique example of the AAOPE strategy is the preparation of multi-enzymes-coencapsulated ZIF-8 that act as enzyme cascade nanoreactors (ECN, Figure 11A). ${ }^{39}$ The coencapsulation of two enzymes, namely, horseradish peroxidise (HRP) and GOx was achieved using AAOPE strategy. Characterization by confocal microscopy clearly proved both HRP 
and GOx are homogenously distributed within ZIF-8 MOF (Figure 11B), which is favourable for biocatalytic cascade reactions. Interestingly, ECN showed around three-fold enhancement in the cascade activity in the formation of glucose than the bulk mixture of the free enzymes in solution (Figure 11C). This enhanced activity of ECN was due to the high population of the substrates at the intercommunicated enzymes in ECN. In addition, ECN was also found to exhibit superior activity for glucose sensing with naked eyes (Figure 11D) than other reported colorimetric glucose sensors. ${ }^{40-44}$

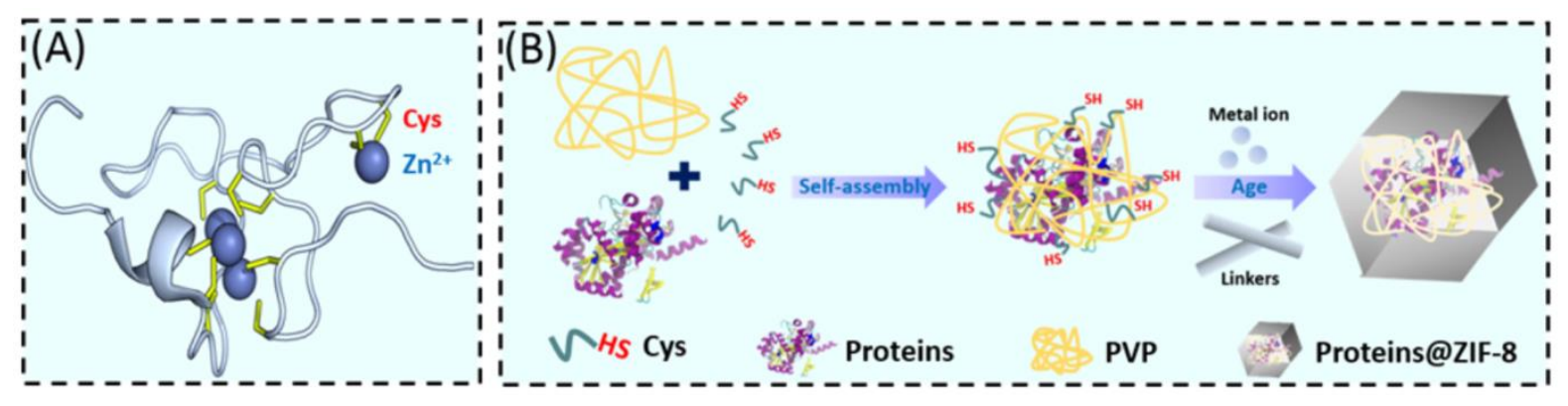

Figure 10. (A) Schematic representation of the $\mathrm{Zn}^{2+-b i n d i n g ~ m e t a l l o t h i o n e i n ; ~(B) ~ S c h e m a t i c ~}$ representation of the amino acid-boosted one pot embedding strategy. Reproduced with permission from ref. ${ }^{37}$ Wiley, Copyright 2019. 


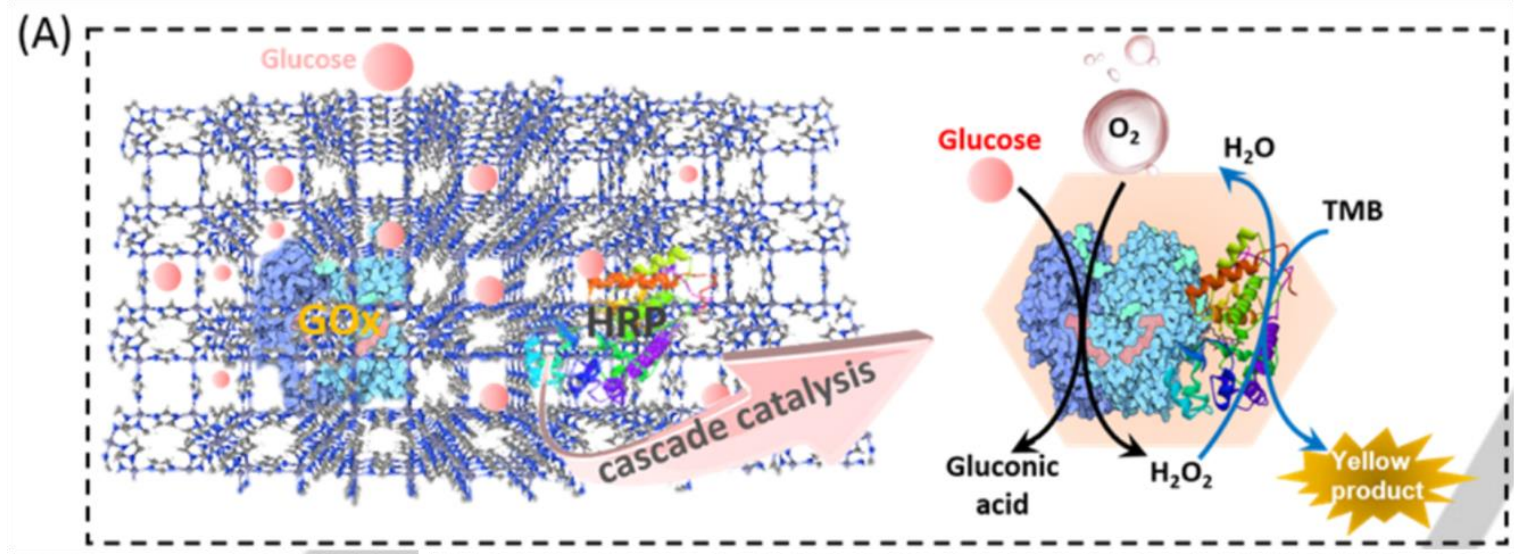

(B)

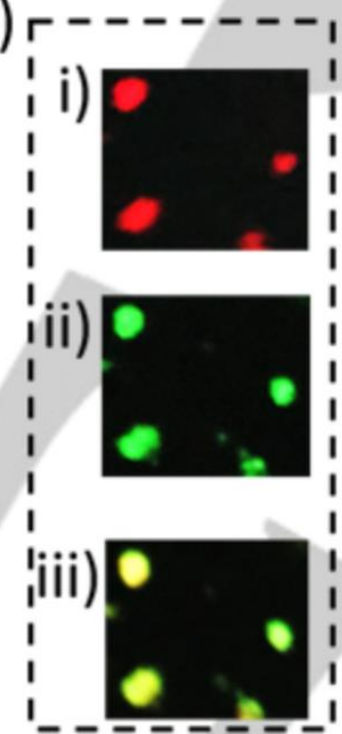

(C)

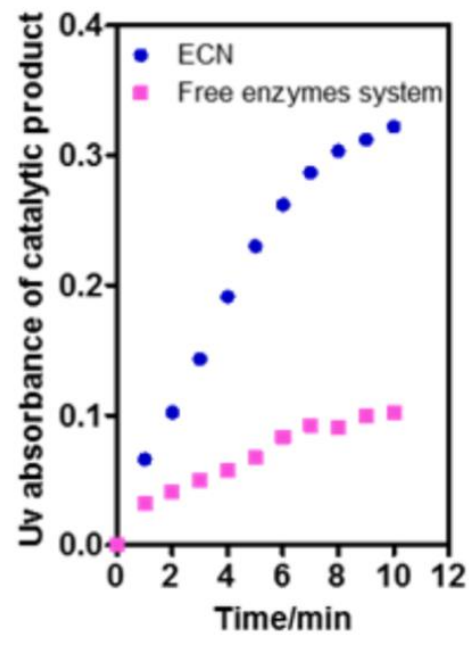

(D)

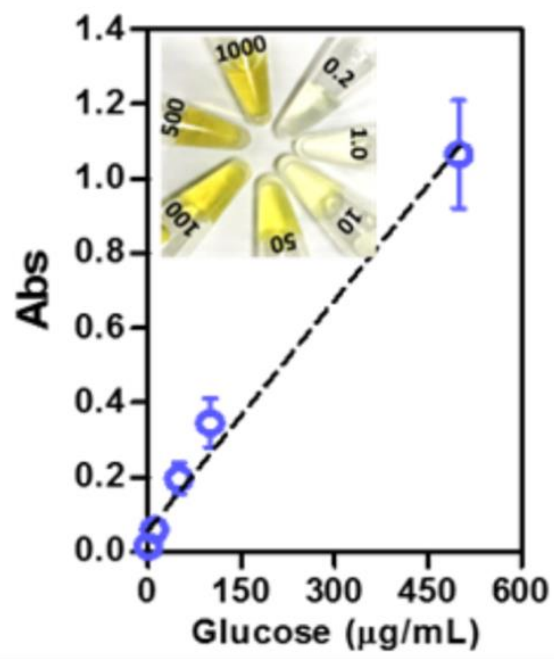

Figure 11. (A) Schematic representation of two-enzyme cascade nanoreactor for catalysis. (B) Confocal microscopy image of the HRP and GOx within ZIF-8; Rhodamine B-labelled HRP at $543 \mathrm{~nm}$ excitation wavelength (i); Fluorescein thioisocyanate-labelled GOx at $488 \mathrm{~nm}$ excitation wavelength (ii); a merged image of the other two images (iii). (C) Monitoring the catalytic product of glucose yielded form ECN versus the free enzymes system by UV absorbance at $450 \mathrm{~nm}$. (D) Linear range of the visual glucose sensing. Reproduced with permission from ref. ${ }^{37}$ Wiley, Copyright 2019.

Recently, HRP and GOx enzymes were cross-linked by rationally designed DNA and later, the multienzyme system was encapsulated into ZIF-8 to obtain GOx\&HRP@DNA/ZIF-8. The activity of this enzymatic complex was tested in the oxidation of glucose followed by ABTS (Figure 12). ${ }^{45}$ Under the optimized reaction conditions, the cascade activity of GOx\&HRP@DNA/ZIF-8 was much superior to that of a mixture of GOx@DNA/ZIF-8 and free HRP, HRP@DNA/ZIF-8 and free GOx, and GOx@DNA/ZIF-8 and HRP@DNA/ZIF-8 under same protein concentration. The enhanced enzymatic activity of GOx\&HRP@DNA/ZIF-8 might be due to rapid consumption of the generated intermediate 
$\mathrm{H}_{2} \mathrm{O}_{2}$ due the proximity of the enzymes. Furthermore, DNA scaffold network provides a good platform for the uniform distribution of GOx and HRP in close proximity within the framework of ZIF-8. The kinetic data revealed that the $\mathrm{K}_{\mathrm{m}}$ and $\mathrm{k}_{\mathrm{cat}} / \mathrm{K}_{\mathrm{m}}$ for GOx\&HRP@DNA/ZIF-8 was $13.8 \mathrm{mM}$ and $3.47 \mathrm{~s}^{-1} \mathrm{mM}^{-1}$, which was 0.27 -fold lower and 9.9-fold higher than those of free GOx\&HRP, respectively, thus suggesting better substrate affinity. In addition, GOx\&HRP@DNA/ZIF-8 showed higher stability than the free enzymes in reusability tests by exhibiting more than $87 \%$ of the initial activity after 14 cycles.

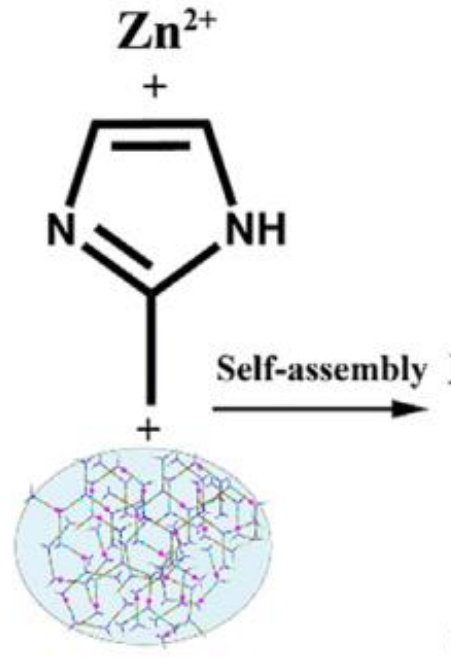

GOx\&HRP@DNA

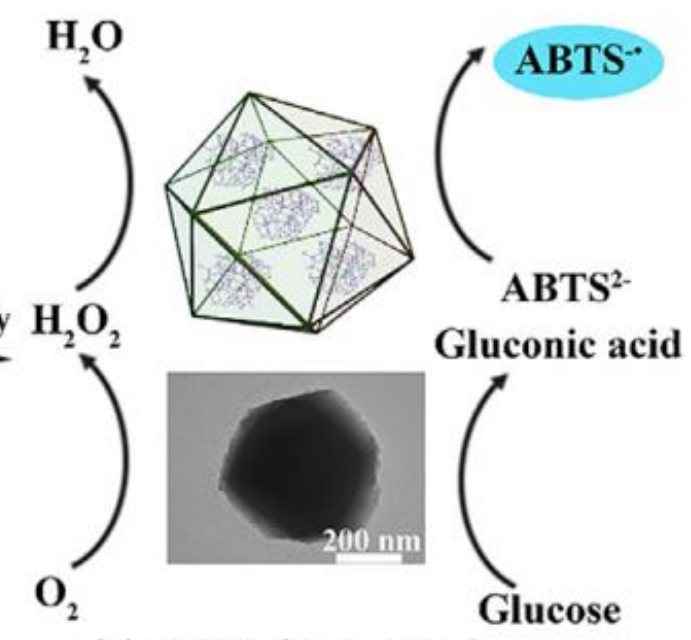

GOx\&HRP@DNA/ZIF-8

Figure 12. Synthesis and cascade catalytic activity of GOx\&HRP@DNA/ZIF-8. Reproduced with permission from ref. ${ }^{45}$ Elsevier, Copyright 2019.

In another work, a cascade bioreactor was constructed by encapsulating HRP and GOx within the pores of ZIF-8 nanostructures (ZIF-8@GOx/HRP) and its catalytic activity was tested in the synergistic cancer starvation/oxidation therapy. ${ }^{46}$ The encapsulated GOx is mainly responsible for the depletion of intratumoral glucose by its transformation into gluconic acid causing the inhibition of the cancer metabolic pathways. Further, the in-situ formed gluconic acid accelerates the reaction between $\mathrm{HRP}$ and the formed $\mathrm{H}_{2} \mathrm{O}_{2}$. Oxidation therapy is based on the ability of $\mathrm{HRP}$ to decompose $\mathrm{H}_{2} \mathrm{O}_{2}$ to give hydroxyl radical as highly reactive oxygen species that generates oxidative stress in the tumor cells causing their death. Interestingly, GOx in ZIF-8@GOx/HRP catalyzed the conversion of glucose to gluconic acid and $\mathrm{H}_{2} \mathrm{O}_{2}$ (Figure 13A), and further, HRP promotes the oxidation of TMB by the in-situ formed $\mathrm{H}_{2} \mathrm{O}_{2}$ providing a glucose-concentration-dependent colour change at $652 \mathrm{~nm}$ (Figure 13B,C), allowing to follow the course of hydroxyl radical production through the cascade reaction. In contrast, 
the free GOx/HRP only exhibited a weak absorption at $652 \mathrm{~nm}$, which is 2.4 -fold lower than the absorption of ZIF-8@GOx/HRP at $100 \mu \mathrm{g} / \mathrm{mL}$. Furthermore, negligible absorption was seen with ZIF8@GOx and ZIF-8@HRP solutions, suggesting that neither GOx nor HRP independently can promote the cascade reaction. These control experiments clearly imply that the as obtained ZIF-8@GOx/HRP could efficiently promote cascade reaction by retaining the enzymatic activities of GOx and HRP and also enhances the catalytic activity of encapsulated enzymes compared to free enzymes. The superior activity of ZIF-8@GOx/HRP is ascribed to the enhanced stability of enzymes by the rigid structure of ZIF-8 as well as their close proximity. In a related theoretical study, it was observed that the small distance between enzymes within the confinement zone accelerates the reaction rates and yield by the faster reaction of the formed intermediates. In contrast, if the intermediate decay were not fast, the rate of cascade reactions would not be altered by the confinement. ${ }^{47}$
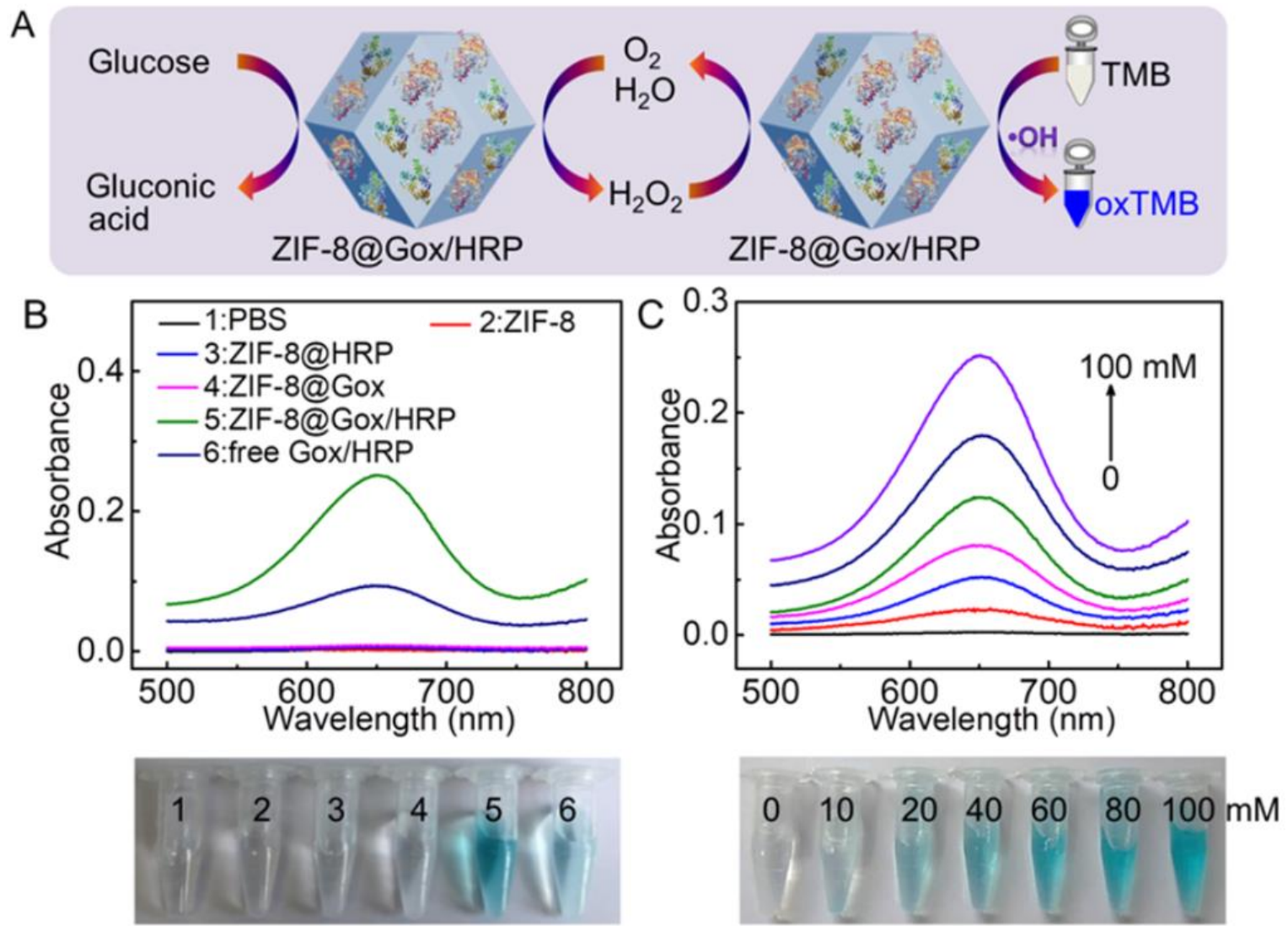

Figure 13. (A) Schematic illustration of cascade catalytic reaction between ZIF-8@GOx/HRP NPs and glucose. (B) Absorbance spectra and visual colour changes of TMB oxidization from cascade catalytic reaction in six different samples. (C) Glucose-concentration-dependent absorbance spectra of TMB 
oxidation from cascade catalytic reaction in ZIF-8@GOx/HRP. Reproduced with permission from ref. ${ }^{46}$ American Chemical Society, Copyright 2019.

A magnetic multi-enzyme solid was fabricated through co-precipitation of enzymes and HKUST-1 (HKUST: Hong Kong University of Science and Technology) precursors with the aid of magnetic $\mathrm{Fe}_{3} \mathrm{O}_{4} \mathrm{NPs}$. The spatial co-localization of GOx and HRP enzymes was achieved using a layerby-layer positional assembly strategy (Figure 14) and the activity of the resulting solids was tested in the cascade oxidation of glucose and o-phenylenediamine. ${ }^{48}$ Among the various solid catalysts employed for this tandem reaction, the use of GOx@HRP@HKUST-1@ $\mathrm{Fe}_{3} \mathrm{O}_{4}$ solid exhibited superior kinetics with a Michaelis constant $\mathrm{K}_{\mathrm{m}}=0.8 \mathrm{mmol} \mathrm{L}^{-1}$ and the maximum reaction rate $\mathrm{V}_{\max }=11.3 \mu \mathrm{mol} \mathrm{L}$ ${ }^{1} \mathrm{~min}^{-1}$. Furthermore, the enzyme-HKUST-1 hybrid showed remarkably operational stability compared to the free enzymes.

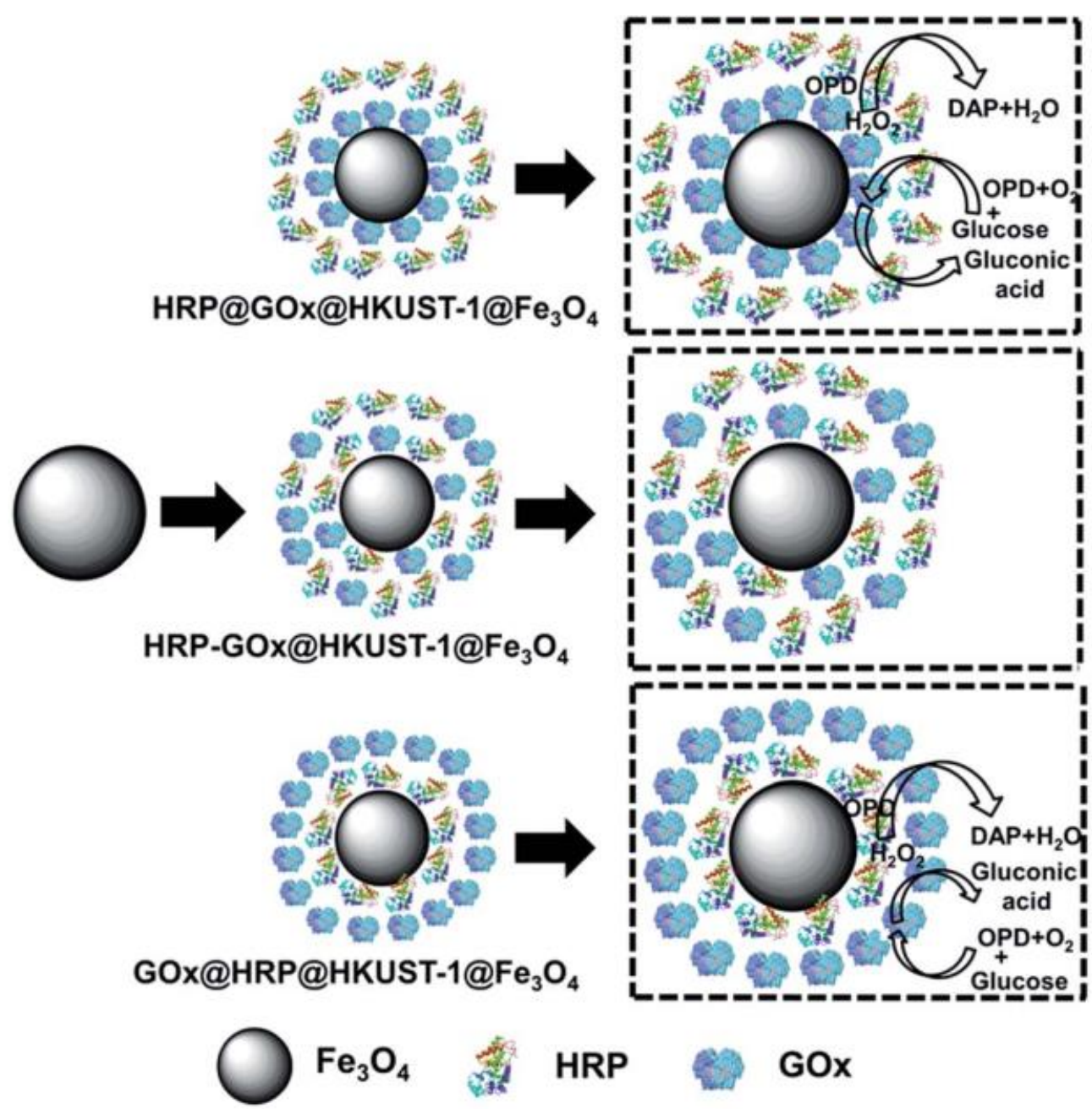


Figure 14. Schematic illustration of the three different strategies for the positional assembly and spatial co-localization of GOx and HRP, and their cascade biocatalysis reaction. DAP: diaminophenazine; OPD: o-phenylenediamine. Reproduced with permission from ref. ${ }^{48}$, Royal Society of Chemistry, Copyright 2017 under Creative Common Licence (CC BY-NC 3.0).

\section{Enzyme@MOFs for cancer therapy}

A dual enzyme-functionalized core-shell nanomotor (designated as UTZCG) based on ZIF-8 MOF NPs was developed for enhancing synergetic photodynamic therapy (PDT) and starvation therapy (ST). ${ }^{49}$ GOx promotes the decomposition of intracellular glucose causing cell starvation and simultaneous generation of $\mathrm{H}_{2} \mathrm{O}_{2}$. On other hand, catalase-triggered decomposition of both endogenous and $\mathrm{GOx}$ generated $\mathrm{H}_{2} \mathrm{O}_{2}$ fuels the nanomotors, increasing their motion and, even resulting in a higher cellular uptake of the therapeutic nanomotors. Meanwhile, the produced oxygen not only promotes singlet oxygen generation during near infrared light-triggered PDT process, but also facilitates the GOxtriggered decomposition of glucose (Figure 15). Overall, this work has demonstrated the possibility to apply MOF based enzymatic systems cancer treatment, the mechanism of the therapeutic action being based on cascade reactions.

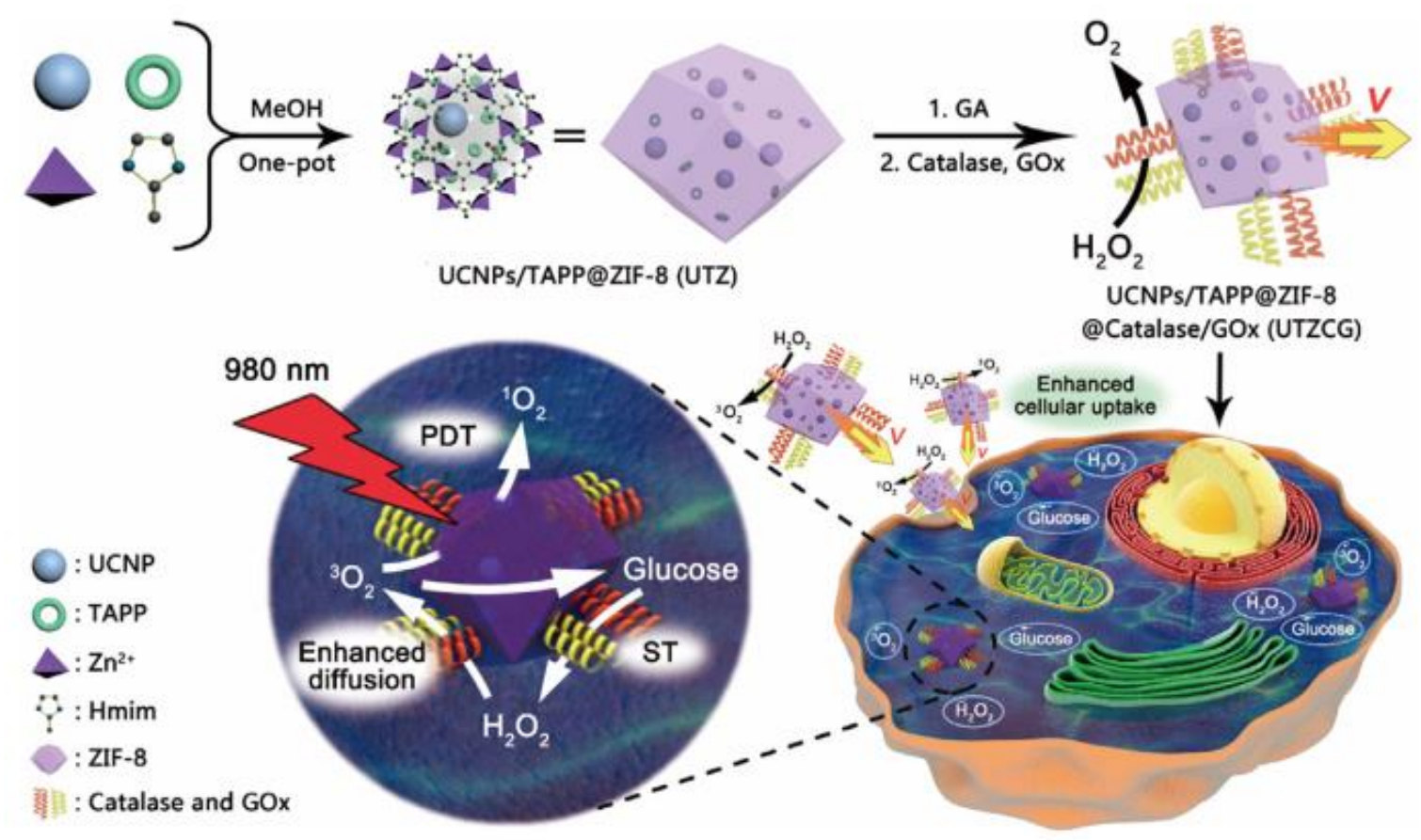


Figure 15. Illustration of the fabrication of a UTZCG nanomotor and synergetic photodynamic-starvation combined anticancer therapy based on the occurrence of cascade reactions. Reproduced with permission from ref. ${ }^{49}$ Elsevier, Copyright 2019.

A highly efficient and sensitive electrochemical impedimetric aptasensor was developed for the detection of carcinoembryonic antigen by using hybrid solid Pt@CuMOFs-hGq-GOx (Figure 16A) as signal transduction probes. ${ }^{50}$ In this solid catalyst, Pt@CuMOFs was utilized as nanocarriers to immobilize Pt NPs, hemin/G-quadruplex (hGq) and GOx. Upon the addition of 3,3-diaminobenzidine (DAB) and glucose to the electrode interface modified with Pt@CuMOFs-hGq-GOx, the cascade reaction was initiated by the oxidation of glucose by GOx by forming, in situ $\mathrm{H}_{2} \mathrm{O}_{2}$. Later, Pt@CuMOFs and $\mathrm{hGq}$ act as synergistic catalysts promoting the oxidation by $\mathrm{H}_{2} \mathrm{O}_{2}$ of $\mathrm{DAB}$ to nonconductive insoluble precipitates (Figure 16B). These results indicate that the close proximity of Pt@CuMOFs, hGq and GOx without having any confinement could also promotes cascade catalysis. These types of results would be highly promising for the development of proteins assaying in clinic medicine due to its high sensitivity and long term stability.
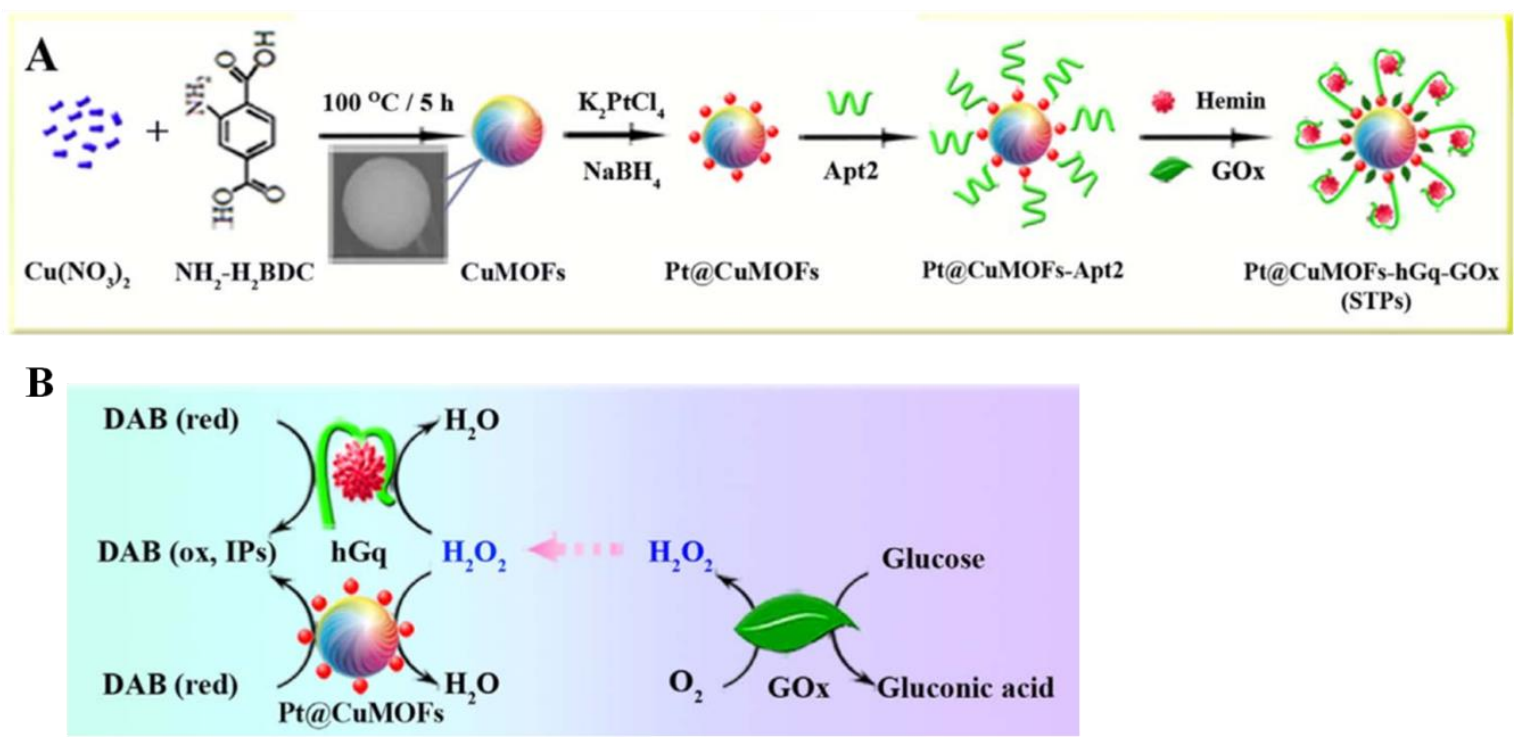

Figure 16. (A) Preparation process of Pt@CuMOFs-hGq-GOx, (B) Cascade catalysis amplification to form nonconductive insoluble precipitates. Reproduced with permission from ref. ${ }^{50}$ Elsevier, Copyright 2017.

\section{Conclusions and Future prospects}


The commented reports have shown that the synthesis of several MOFs, their composition and structure is compatible with enzymes that become embedded inside or strongly adsorbed on the MOF crystallites without losing their catalytic activity. In fact, there are several examples that have shown that the intrinsic catalytic activity of the enzyme even increases, particularly for cascade reactions, as consequence of the association with complementary catalytic sites in close proximity. It is claimed that this proximity is a key factor in the enhancement observed of the enzyme-MOF assembly in cascade reaction. Furthermore, in addition to increased activity, it appears that the enzyme becomes stabilized when it is confined or associated to the MOF. In addition the enzyme-MOF system can be easily recovered or immobilized.

It can be anticipated that the area will expand with additional examples of enzymes, particularly those commercially available. It has been shown that the enzyme does not need to be occluded within mesopores, but many types of MOF particles can grow around the enzyme, without blocking accessibility of substrates and reagents to the enzyme active centre. This possibility expands also considerably the type of MOFs that can be used, with the main limitation that the synthesis conditions have to be compatible with the enzyme stability.

Thus, considering that enzyme immobilization has been long pursued as a way to implement biocatalysis at commercial level, the available data indicate that MOFs are among the best candidates as supports with the additional advantage that MOFs can introduce Lewis acid/base centres and can host metal and metal oxide NPs. All these features, together with structural stability, will be exploited in the near future to develop cascade reactions, combining two or more elementary reactions in a single process.

\section{Acknowledgements}

Financial support by the Spanish Ministry of Science and Innovation (Severo Ochoa and CTQ2018980237-CO2-1 and Generalitat Valenciana (Prometeo 2017-083) is gratefully acknowledged. A.D. thanks the University Grants Commission (UGC), New Delhi, for the award of an Assistant Professorship under its Faculty Recharge Programme. A.D. also thanks the Department of Science and Technology, India, for the financial support through Extra Mural Research Funding (EMR/2016/006500). 


\section{Conflict of Interests}

Authors declare no conflict of interests

\section{References}

1. D. E. Koshland, Proc. Natl. Acad. Sci. U.S.A., 1958, 44, 98-104.

2. R. V. Stanton, M. Perakyla, D. Bakowies and P. A. Kollman, J. Am. Chem. Soc., 1998, 120, 34483457.

3. B. Kuhn and P. A. Kollman, J. Am. Chem. Soc., 2000, 122, 2586-2596.

4. T. C. Bruice and F. C. Lightstone, Acc. Chem. Res., 1999, 32, 127-136.

5. S. Tsitkov and H. Hess, ACS Catal., 2019, 9, 2432-2439.

6. J. Muschiol, C. Peters, N. Oberleitner, M. D. Mihovilovic, U. T. Bornscheuer and F. Rudroff, Chem. Commun., 2015, 51, 5798-5811.

7. Z. Liu, Y. Lv and Z. An, Angew. Chem. Int. Ed., 2017, 56, 13852-13856.

8. H. Wang, L. Cheng, S. Ma, L. Ding, W. Zhang, Z. Xu, D. Li and L. Gao, ACS Appl. Mater. Interfaces 2020, 12, 20191-20201.

9. M. B. Majewski, A. J. Howarth, P. Li, M. R. Wasielewski, J. T. Hupp and O. K. Farha, CrystEngComm, 2017, 19, 4082-4091.

10. L. Chen and Q. Xu, Matter 2019, 1, 57-89.

11. X. Lian, Y. Fang, E. Joseph, Q. Wang, J. Li, S. Banerjee, C. Lollar, X. Wang and H.-C. Zhou, Chem. Soc. Rev., 2017, 46, 3386-3401.

12. R. J. Drout, L. Robison and O. K. Farha, Coord. Chem. Rev., 2019, 381, 151-160.

13. T.-H. Wei, S.-H. Wu, Y.-D. Huang, W.-S. Lo, B. P. Williams, S.-Y. Chen, H.-C. Yang, Y.-S. Hsu, Z.Y. Lin, X.-H. Chen, P. E. Kuo, L.-Y. Chou, Tsung C.-K. and F.-K. Shieh, Nature Commun., 2019, 10,5002

14. S. Zhang, M. Du, P. Shao, L. Wang, J. Ye, J. Chen and J. Chen, Environ. Sci. Technol. , 2018, 52, 12708-12716.

15. D. Farrusseng, S. Aguado and C. Pinel, Angew. Chem., Int. Ed., 2009, 48, 7502-7513.

16. L. Zhu, X.-Q. Liu, H.-L. Jiang and L.-B. Sun, Chem. Rev., 2017, 117, 8129-8176.

17. A. Dhakshinamoorthy, Z. Li and H. Garcia, Chem. Soc. Rev., 2018, 47, 8134-8172.

18. O. K. Farha, I. Eryazici, N. C. Jeong, B. G. Hauser, C. E. Wilmer, A. A. Sarjeant, R. Q. Snurr, S. T. Nguyen, A. O. Yazaydın and J. T. Hupp, J. Am. Chem. Soc., 2012, 134, 15016-15021.

19. R. L. Martin and M. Haranczyk, Chem. Sci., 2013, 4, 1781-1785.

20. Y. He, B. Li, M. O'Keeffe and B. Chen, Chem. Soc. Rev., 2014, 43, 5618-5656.

21. S. Yuan, L. Feng, K. Wang, J. Pang, M. Bosch, C. Lollar, Y. Sun, J. Qin, X. Yang, P. Zhang, Q. Wang, L. Zou, Y. Zhang, L. Zhang, Y. Fang, J. Li and H.-C. Zhou, Adv. Mater., 2018, 30, 1704303.

22. A. Dhakshinamoorthy, S. Navalon, A. M. Asiri and H. Garcia, Chem. Commun., 2020, 56, 26-45.

23. A. Dhakshinamoorthy, A. Santiago-Portillo, A. M. Asiri and H. Garcia, ChemCatChem, 2019, 11, 899-923.

24. A. Dhakshinamoorthy, A. M. Asiri and H. Garcia, Adv. Mater., 2019, 31, 1900617.

25. E. Gkaniatsou, C. Sicard, R. Ricoux, J.-P. Mahy, N. Steunou and C. Serre, Mater. Horiz., 2017, 4, 55-63.

26. X. Cai, M. Zhang, W. Wei, Y. Zhang, Z. Wang and J. Zheng, Biotech. Lett., 2020, 42, 269-276.

27. C. Lin, K. Xu, R. Zheng and Y. Zheng, Chem. Commun., 2019, 55, 5697-5700.

28. S. S. Nadar, L. Vaidya and V. K. Rathod, Int. J. Biol. Macromol., 2020, 149, 861-876.

29. S. Dutta, N. Kumari, S. Dubbu, S. W. Jang, A. Kumar, H. Ohtsu, J. Kim, S. H. Cho, M. Kawano and I. S. Lee, Angew. Chem. Int. Ed., 2020, 59, 3416-3422.

30. Y. Wang, N. Zhang, E. Zhang, Y. Han, Z. Qi, M. B. Ansorge-Schumacher, Y. Ge and C. Wu, Chem. Eur. J., 2019, 25, 1716-1721. 
31. T. Li, H. Qiu, N. Liu, J. Li, Y. Bao and W. Tong, Colloids Surfaces B: Biointerfaces, 2020, 191, 111001.

32. W. Xu, L. Jiao, H. Yan, Y. Wu, L. Chen, W. Gu, D. Du, Y. Lin and C. Zhu, ACS Appl. Mater. Interfaces, 2019, 11, 22096-22101.

33. W. Tan, T. Wei, J. Huo, M. Loubidi, T. Liu, Y. Liang and L. Deng, ACS Appl. Mater. Interfaces, 2019, 11, 36782-36788.

34. X. Liu, W. Qi, Y. Wang, D. Lin, X. Yang, R. Su and Z. He, ACS Appl. Mater. Interfaces, 2018, 10, 33407-33415.

35. X. Zhong, H. Xia, W. Huang, Z. Li and Y. Jiang, Chem. Eng. J., 2020, 381122758.

36. M. B. Quin, K. K. Wallin, G. Zhanga and C. Schmidt-Dannert, Org. Biomol. Chem., 2017, 15, 4260-4271.

37. C. Guosheng, H. Siming, K. Xiaoxue, W. Songbo, H. Shuyao, J. Shuqi, S. Jun, Z. Fang and O. Gangfeng, Angew. Chem. Int. Ed., 2019, 58, 1463-1467.

38. R. D. Palmiter, Proc. Natl. Acad. Sci. U. S. A., 1998, 95, 8428-8430.

39. W.-H. Chen, M. Vázquez-González, A. Zoabi, R. A. Reziq and I. Willner Nat. Catal., 2018, 1, 689695.

40. J. Mu, Y. Wang, M. Zhao and L. Zhang, Chem. Commun., 2012, 48, 2540.

41. Y. Song, K. Qu, C. Zhao, J. Ren and X. Qu, Adv. Mater., 2010, 22, 2206.

42. H. Wei and E. Wang, Anal. Chem., 2008, 80, 2250.

43. Q. Yan, B. Peng, G. Su, B. E. Cohan, T. C. Major and M. E. Meyerhoff, Anal. Chem., 2011, 83, 8341.

44. X. Wu, J. Ge, C. Yang, M. Hou and Z. Liu, Chem. Commun., 2015, 51, 13408-13411.

45. J. Song, W. He, H. Shen, Z. Zhou, M. Li, P. Su and Y. Yang, Chem. Eng. J., 2019, 363, 174-182.

46. J. Bai, C. Peng, L. Guo and M. Zhou, ACS Biomater. Sci. Eng., 2019, 5, 6207-6215.

47. Y. Cao, X. Li, J. Xiong, L. Wang, L.-T. Yan and J. Ge, Nanoscale, 2019, 11, 22108-22117.

48. S. Chen, L. Wen, F. Svec, T. Tan and Y. Lv, RSC Adv., 2017, 7, 21205-21213.

49. Y. You, D. Xu, X. Pan and X. Ma, Appl. Mater. Today, 2019, 16, 508-517.

50. X. Zhou, S. Guo, J. Gao, J. Zhao, S. Xue and W. Xu, Biosensors Bioelectronics, 2017, 98, 83-90. 


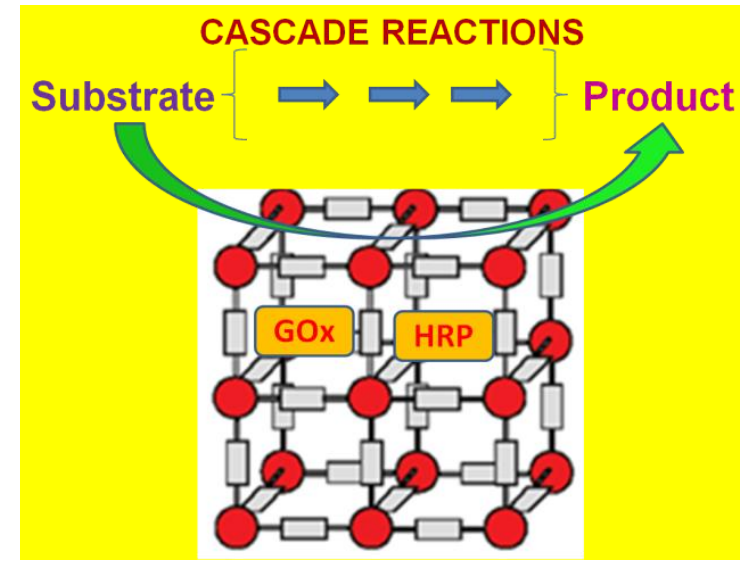

This work describes the recent developments for the integration of enzymes with metal organic frameworks as multifunctional solids for cascade (bio)catalysis. 